\title{
Paternal treatment barriers predictability of preference for types of postpartum depression
}

\author{
Pooja Sohal ${ }^{1}$, Emily Cameron ${ }^{1}$, Lianne M. Tomfohr-Madsen ${ }^{1,2,3}$
}

${ }^{1}$ Department of Psychology, University of Calgary, Calgary, AB, Canada

${ }^{2}$ Alberta Children's Hospital Research Institute for Child and Maternal Health (ACHRI), Calgary, AB, Canada

${ }^{3}$ Department of Pediatrics, Alberta Children's Hospital, Calgary, AB, Canada

\section{Abstract}

There is a growing prevalence of paternal postpartum depression (PPD) but many individuals fail to seek treatment due to some form of inconvenience or receiving treatment outside their first choice of treatment. Recent research has shown that identifying specific preferences to types of treatment encourage fathers to continue treatment and improve depression outcome. The objective of this study is to explore treatment barriers of PPD within health care systems to further improve treatment outcomes and to provide more accessible therapy. Fathers of infants aged 0-12 months were recruited from low-risk maternity clinics, baby shows and partner referrals. Participants $(N=140)$ completed a 20 -minute survey upon recruitment. Surveys contained measurements of barriers to treatment and preferences to broad treatment categories for paternal PPD including pharmacotherapy, couple therapy and individual therapy. Correlation analyses and multinomial logistic regression using pharmacotherapy as the reference group was conducted to examine if specific types of barriers could predict types of treatments for depression in fathers. Correlation analyses indicated that three specific barriers were significantly related to treatment preference; specifically, participants' responses indicated that barriers included that prayer would be enough in helping to treat depression $(r=.21, p$ $=.011)$, the depression would go away once the baby is a little older $(r=.18, p=.033)$, and professional mental health services would not be sensitive to the participants' race, ethnicity or culture $(r=.19, p=.022)$. One broad category of treatment barriers, Spiritual Barriers, was also significantly related to treatment preference $(r=.19, p=.023)$. Multinomial regression models were significant for the three significant individual barriers $(X 2=23.01, p=.001)$ and Spiritual Barriers $(X 2=16.54, p<.001)$ in predicting likelihood for treatment preference. The results of the current study indicated that barriers to seek professional therapies included spirituality, beliefs that the depression would remit overtime, and concerns over professionals' responses to demographic characteristics. Future research should focus on addressing the barriers to treatment to promote mental health treatment for new fathers.

Cite as: Sohal P., Cameron E., Tomfohr-Madsen LM. 2019. Paternal treatment barriers predictability of preference for types of postpartum depression. Alberta Academic Review, Vol 2 (3) 3, CASCH Special Issue (not peer-reviewed), DOI: 10.29173/aar91.

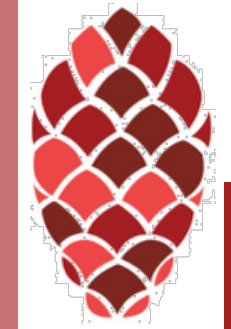

